\title{
The role of drugs and selected dietary factors in cutaneous squamous cell carcinogenesis
}

\author{
Tatsiana Damps¹, Joanna Czuwara², Olga Warszawik-Hendzel², Aleksandra Misicka ${ }^{1}$, Lidia Rudnicka² \\ ${ }^{1}$ Department of Neuropeptides, Mossakowski Medical Research Centre, Polish Academy of Sciences, Warsaw, Poland \\ ${ }^{2}$ Department of Dermatology, Medical University of Warsaw, Warsaw, Poland \\ Adv Dermatol Allergol 2021; XXXVIII (2): 198-204 \\ DOI: https://doi.org/10.5114/ada.2021.106196
}

\begin{abstract}
Cutaneous squamous cell carcinoma represents the second most common non-melanoma skin cancer and its incidence increases worldwide. This review provides an overview of selected exogenous risk factors for cutaneous squamous cell carcinoma, which include drugs (azathioprine, calcineurin inhibitors, hydrochlorothiazide, angiotensinconverting-enzyme inhibitors) and few dietary factors (fat meet, whole milk products, arsenic) to better understand squamous skin cancer etiopathogenesis. Ingredients such as leafy vegetables, nuts, fish, caffeine, niacin are preventive factors for cutaneous squamous cell cancer. The heart transplant recipients have an increased risk of squamous cell carcinoma development than kidney or liver transplant ones and switching photosensitizing azathioprine to mycophenolate mofetil can reduce the incidence of cutaneous squamous cell carcinoma. The great attention should be paid to early change of cardiac photosensitizing antihypertensive drugs to non-photosensitizing ones among patients with a history of prior skin cancers and among organ transplant recipients. Based on current knowledge that ultra-violet radiation is the main risk factor for squamous cell carcinoma development, promotion of the skin self-examination, photoprotection, tanning bed avoidance and early skin cancer diagnosis is important for this tumour prevention.
\end{abstract}

Key words: squamous cell carcinoma, skin cancer, risk factors.

\section{Introduction}

Cutaneous squamous cell carcinoma (CSCC) belongs to non-melanoma skin cancers (NMSC) defined as the proliferation of atypical neoplastic interfollicular epidermal suprabasal keratinocytes. Almost $20 \%$ of NMSC are cutaneous SCC and the incidence of CSCC rises due to intensifying ultraviolet (UV) exposure, advancing population age, enhanced public awareness of skin cancer screening and more frequent skin examinations by physicians [1-4].

One of the highest incidence rates of skin cancer exists in Australia. Recently, Pandeya et al. [5] reported the person-based incidence of keratinocyte cancer excisions in Australia. It was 1531 per 100000 person-years, and the incidence increased with age with the predominance of men (standardized incidence ratio $=1.43 ; 95 \% \mathrm{Cl}: 1.42-$ 1.45). Keratinocyte cancer incidence was 8 times higher among people with a past history of excisions than in healthy population [5].
Muzic et al. [6] in his retrospective study showed that the incidence of cSCC in residents of Olmsted County, Minnesota, between 2000 and 2010 was rising as compared with the results of previous population-based studies. In 1653 patients with the mean age of 70.5 years, 1711 cases of cSCC were diagnosed with the predominance of men $(55.1 \%)$ over women $(44.9 \%)$ in the population of Olmsted County, Minnesota. Furthermore, in patients younger than 40 years, the incidence rates for cSCC were higher in younger women than in younger men in this cohort study [6].

The main risk factors for cSCC development include chronic UV radiation, therapeutic UV light exposure, immunosuppression (especially in organ transplant recipients), exposure to carcinogenic chemicals, drugs, cigarette smoking, chronic skin ulcerations, HPV infections, Fitzpatrick type I or II skin characteristics [1, 3, 4, 7].

This article aims to overview exogenous risk factors for cSCC with the consideration of the main preventive method application. The analysis of previously published

Address for correspondence: Joanna Czuwara MD, PhD, Department of Dermatology, Medical University of Warsaw, 82 A Koszykowa St, Warsaw 02-008, Poland, phone: +48 22502 13 11, e-mail: jczuwara@yahoo.com Received: 3.10.2019, accepted: 20.02.2020. 
studies about squamous carcinoma of the skin was performed. The literature overview was carried out by searching the PubMed, Scopus and EBSCO databases, complemented by a thorough search of references focusing on the last 15 years of publication.

\section{Risk factors of cSCC development \\ Drugs}

\section{Long-term immunosuppressive medications in transplant recipients}

Patients treated with immunosuppressive drugs form a group with the highest risk of SCC development and this skin tumour is the most common in immunocompromised patients. Compared with the general population, solid organ transplant recipients have a 65 to 250 times higher risk of developing SCC, which may be more aggressive than in immunocompetent individuals [7-9]. According to data from the national renal transplant database and the national cancer registry in Ireland, the number of renal transplant recipients with skin cancers and other malignancies was analysed by Moloney et al. (1558 patients, 64\% males). Most patients were on triple immunosuppression with cyclosporine, azathioprine and steroids. The authors showed the biphasic increase in skin cancer incidence following renal transplantation, determined by the age: at the age under 50 years reaching 200 times the risk for an age-matched non-transplanted population by 6 year post-transplant. The risk of invasive SCC increases 82-fold in comparison to non-transplanted population [9].

Collett et al. [10] conducted a study in 37617 transplant recipients in the United Kingdom with the predominance of kidney transplant recipients. A total of 5706 (15\%) patients developed a cancer over 16 years of follow-up. In 3276 patients (8.7\% of this population) the first cancer registered following transplantation was nonmelanoma skin cancer. Moreover, the authors evaluated that organ recipients have more than double the 10-year incidence rate for de novo cancers. The risk of NMSC occurring in transplant recipients is over 13 times higher than of the general population [10].

The authors of population-based, case-control study involved 95 kidney transplant recipients who had contracted cSCC after the transplantation and control population of 154 kidney transplanted patients without a skin SCC history, showing that patients with light blond/red hair colour had a higher odds ratio for SCC than those with dark hair, 3.2 (95\% Cl: 1.2-8.2), and patients with warts after the transplantation had a higher odds ratio for SCC than those without warts, 2.2 (95\% Cl: 1.2-4.2) [11]. According to the authors of this study, Lindelöf et al., poor tanning ability rather than the amount of sun exposure is associated with the development of CSCC in kid- ney transplant recipients and warts appearing after the transplantation indicate an increased risk of CSCC [11].

In regard to 3393 adult patients after heart transplantation in Spain, the incidence rates of SCC and basal cell carcinoma (BCC) were 8.5 and 5.2 per 1000 persons/ year, respectively [12]. The majority (84.7\%) of patients were men and median follow-up was 5.2 years. In this study 204 patients developed 324 skin cancers, in which SCC comprised $54.2 \%$ and BCC $33.3 \%$ of cases. The incidence of NMSC increased from 4.4 per 1000 persons/ year among patients aged $<45$ years at heart transplantation to 25.5 among patients aged $>65$. The results of this study showed that mycophenolate mofetil and tacrolimus carried a reduced risk of CSCC, azathioprine increased the risk of cSCC and cyclosporine was neutral [12]. The relative risk of mycophenolate mofetil was 0.3 (0.2-0.6; $p<0.0005)$ and azathioprine was 1.8 (1.2-2.7; $p<0.0032)$ for SCC.

The cause of azathioprine influence on SCC development may be associated with its photosensitizing effect and increased accumulation of 6-thioguanine in DNA [13]. Interestingly, Atkar et al. [14] presented population studies of French and British organ transplant patients implicating solar UVA in their increased skin cancer risk. Facial tumours in French patients arose predominantly on the left side, those in British patients on the right side of the face. This is due to the role of UVA penetration via glass-filtered UV (UVB does not penetrate glass whereas UVA does) when driving [14].

Other data regarding influence of mycophenolate mofetil on SCC development were obtained in a study by Vos et al. [15] among 544 patients from a Dutch lung transplant recipients (LTR) cohort with a median survival of 11.05 years, 52 (9.6\%) LTRs developed at least one cSCC, with a cumulative incidence of $3.9 \%$ and $15.3 \%$ after 5 and 10 years, respectively. Multivariate analyses showed that the sequential use of azathioprine and mycophenolate mofetil was associated with a lower risk of cSCC development (hazard ratio $=0.24 ; 95 \% \mathrm{Cl}$ : 0.100.56) compared with azathioprine use only. The authors of this study found that switching from azathioprine to mycophenolate mofetil was associated with a reduced incidence of cSCC in lung transplant recipients [15].

To summarize, the heart transplant recipients, getting a higher amount of immunosuppression (usually prednisone, azathioprine and cyclosporine), have an increased risk of SCC development than kidney or liver transplant recipients [16-18]. Azathioprine, a purine antimetabolite immunosuppressant, photosensitizes the skin and causes the production of mutagenic reactive oxygen species leading to an increased risk of skin SCC [19]. Switching from azathioprine to mycophenolate mofetil could reduce the incidence of cSCC [20].

Preventive measures assume great importance in post-transplant patients with special attention devoted to older male patients with a long-term follow up [21-23]. 
A lower dose of immunosuppression could decrease the incidence of SCC without a significant effect on the risk of organ rejection, moreover in patients with an aggressive course of SCCs following organ transplant, alteration of immunosuppressive treatment should be considered [23]. Patients have to be educated about the risk factors for SCCs and regular skin examination once a year at least. Patients with in situ carcinoma (actinic keratosis, Bowen's disease) should be followed up more frequently, at least twice a year.

\section{Cardiac drugs}

Many cardiac drugs photosensitize, heightening the reactivity of the skin to sunlight. Photosensitizing antihypertensive drugs have been associated with lip cancer and some of them with cutaneous SCC, including $\alpha 2$ receptor agonists and diuretics, such as loop, potassium-sparing, thiazide, and combination drugs [24, 25]. An association between diuretic hydrochlorothiazide use and SCC of the lip was reported by Pottegard et al. [24] who noted a clear dose-response pattern, with an estimated 7-fold increased risk of lip SCC with the cumulative use of $100000 \mathrm{mg}$ or more of hydrochlorothiazide. Similar results were shown by authors from the United States, where hydrochlorothiazide is one of the most widely used drug, as well as in Western Europe [26, 27].

The Danish Cancer Registry shows that high use of hydrochlorothiazide ( $\geq 50000 \mathrm{mg}$ ) was associated with the odds ratio (OR) of 3.98 (95\% Cl: 3.68-4.31) for SCC in patients with non-melanoma skin cancer identified in 2004-2012. The authors of this study showed a clear dose-response relationship between hydrochlorothiazide use and SCC [28]. It does not mean that hydrochlorothiazide should be forbidden for each patient, because it does not have a direct carcinogenetic impact, but indirectly leads to increased skin photosensitivity. The regular use of creams with high sun protection factor (SPF) is recommended and patients should be under dermoscopic control and observation of nevi presenting doubtful dermoscopic neoplastic features, especially among patients with a history of immunosuppression and chronic UV radiation exposure.

Other cardiac drugs such as angiotensin-convertingenzyme inhibitors (ACEIs) (captopril, enalapril, lisinopril, ramipril) and angiotensin-receptor blockers (ARBs) (in particular losartan), present photosensitivity as a potential adverse effect. In the matched cohort study by Nardone et al. [29] including patients with a documented order for an ACEIs and ARBs with no prior history of skin cancer, detected 182 cases of SCC among the 27134 patients exposed to ACEI, and 106 cases of SCC among the 13818 patients exposed to ARB. The authors of this interesting study showed that a selective stimulation of type 2 angiotensin-receptors promotes angiogenesis, and thus cancer progression [29].
Photosensitizing drugs absorb energy from UV-radiation causing the generation of reactive oxygen species which damage DNA and produce inflammation. In conclusion, hydrochlorothiazide, hydrochlorothiazide combination medications, ACEls, losartan cause a dose-dependent increased risk of SCC [26, 27, 29]. An increased awareness and education, especially for patients who are at high risk for skin cancer, is required.

\section{Non-steroidal anti-inflammatory drugs (NSAIDs)}

A few epidemiological studies conducted in recent years suggested that non-steroidal anti-inflammatory drugs (NSAIDs) known for many years as anti-inflammatory, analgesic and antipyretic medications may also decrease the risk of cancer development. The mechanism of this phenomenon is based on the inhibition of cyclooxygenase enzyme (COX), an inhibitor of apoptosis and stimulator of the process of invasion and tumour angiogenesis. Numerous studies confirmed the hypothesis of the role of NSAIDs as chemoprotective agents against skin malignancies such as actinic keratosis, SCC, BCC and melanoma. Johannesdottir et al. conducted a study in 1974 on patients diagnosed with cSCC, 13316 patients with BCC and 3242 cases with melanoma [30]. The authors demonstrated that the risk of cSCC is reduced around $15 \%$ in patients taking NSAIDs compared to patients who do not take them. Also they found that reduced risk correlated with longer treatment duration.

A population-based case-control analysis was performed using the Clinical Practice Research Datalink: 7864 patients with a history of SCC diagnosed between 1995 and 2013 were identified and matched 4 NMSC-free controls to SCC case, respectively, on age, sex, general practice, calendar time and years of history in the database. Prior NSAID exposure was compared between cases and controls using multivariate conditional logistic regression analyses controlling for several potential confounders. The risk of SCC was decreased in regular users of any NSAIDs (adj. OR $=0.89,95 \% \mathrm{Cl}$ : 0.82-0.97), with the strongest risk reduction observed in current users of COX inhibitors (coxibs) (adj. OR $=0.77,95 \% \mathrm{Cl}$ : 0.62-0.95) [31]. To conclude, data of nine recently published studies (five case-control, three cohort, and one intervention study) showed significantly reduced risks of SCC among users of non-aspirin NSAIDs (relative risk $(R R)=0.85,95 \% \mathrm{Cl}: 0.78-0.94)$ and among users of any NSAIDS (RR $=0.82,95 \% \mathrm{Cl}: 0.71-0.94)$ compared with nonusers with the effect seen particularly in those with previous actinic skin tumours [32].

Another study prospectively examined whether regular, inconsistent, or no/low-use of NSAIDs is associated with a lower NMSC risk among 54728 postmenopausal Caucasian women in the Women's Health Initiative Observational Study. The authors showed that there was no association between regular NSAID-use and NMSC risk relative to no/low-users. However, in a subgroup analy- 
sis of 5325 women with a history of skin cancer, odds of NMSC were lower among regular NSAID users compared to no/low-users. Inconsistent NSAID use and acetaminophen use were not associated with the NMSC risk [33].

Recently, Pandeya et al. observed no association between NSAID or aspirin use and the risk of SCC among average to low risk participants among 1470 patients after a median of 3 years of follow up. Aspirin use was associated with a reduced risk of SCC (HR $=0.77,95 \% \mathrm{Cl}$ : 0.64-0.93) only among infrequent users [34].

While some weak inverse associations were observed between prior use of aspirin or NSAIDs and skin cancer, further studies are needed to confirm these correlations.

\section{Diet}

There are some publications showing a strong link between dietary factors and the risk of cSCC development. In a prospective community-based study of Australian adults, Hughes et al. estimated that green leafy vegetables possess anticancer properties against SCC. They used answers from a validated food frequency questionnaire of 1056 randomly selected adults living in an Australian sub-tropical community. The authors of the publication provided evidence that intake of green leafy vegetables such as spinach, lettuce, silver beet in persons with a history of skin cancer is associated with a decreased incidence of SCC (RR $=0.45$, 95\% Cl: 0.22-0.91; $p$ for trend $=0.02$ ), while high intakes of unmodified dairy products such as whole milk, cheese and yoghurt are associated with an increased risk of SCC $(\mathrm{RR}=2.53,95 \% \mathrm{Cl}: 1.15-5.54$; $p$ for trend $=$ 0.03). There were no associations among those patients without prior skin cancer history [35].

Another dietary factor with anticancer properties is caffeine, even in its most recognizable form in tea and coffee. Caffeine has the ability to induce apoptosis in sun-damaged keratinocytes. Thus, it exerts a protective effect against UV-induced carcinogenesis. To confirm this statement, Abel et al. conducted a study among 93676 Caucasian women with non-melanoma skin cancer. The prevalence of NMSC in women who consumed six or more cups of caffeinated coffee daily was reduced by $30 \%$. The prevalence was dose-dependent. In contrast to caffeinated coffee, daily consumption of decaffeinated coffee was not associated with a significant change in self-reported non-melanoma skin cancer [36].

Pothiawala et al. conducted a cohort study including 143129 US Caucasians and observed the incidence of melanoma, SCC and BCC in participants having different body mass index (BMI). The authors claimed that BMI in the obese range was associated with a $32 \%$ lower risk of developing SCC, and those with a BMI in the morbidly obese category - with a 37\% lower risk of developing SCC than in the general population. It was the first prospective study investigating the relationship between $\mathrm{BMI}$ and both melanoma and NMSC indicating that obesity is associated with a decreased risk of NMSC. The reduced risk of SCC among the obese is connected with the fact that obese individuals were shown to have lower levels of physical activity outdoors, which translated into less cumulative lifetime sun exposure - the main risk factor for cSCC. This is not per se the protective effect of obesity and, consequently, BMI on SCC incidence and morbidity [37].

A high intake of meat and fat could increase the risk of developing SCC [38]. Ibiebele et al. undertook an investigation into the association between dietary fat and BCC and SCC, using data collected over 11 years of a prospective study of Australian adults from the community of Nambour, Australia. They took a full account of sunexposure histories and other established risk factors for skin cancers. The authors showed no significant linear trends in the overall risk of BCC or SCC of the skin with increasing total fat intake [38]. However, in participants with a history of skin cancer, total fat intake (multivariable adjusted $R R=2.42,95 \% \mathrm{Cl}: 1.20-4.88 ; p$ for trend $=$ $0.01)$ was associated with an increased number of SCC tumours comparing the highest and lowest tertile. In conclusion, SCC tumour risk increased as total fat intake increased in people with a history of skin cancer. In 2017, Park et al. reported a potential protective role of niacin intake on SCC development, but there was no beneficial role of niacin on BCC or melanoma incidence [39]. Based on 72308 women and 41808 men, niacin intake was assessed every 2 to 4 years during follow-up and cumulative averaged intake. The authors documented 2530 cases of SCC and total niacin intake was inversely associated with SCC risk (95\% Cl: 0.74-0.95; $p$ for trend $=0.08$ ) [39].

Rice consumption could also be a risk factor for SCC development, because it may be an important source of exposure to arsenic (As). Arsenic potentiates other carcinogenetic factors, mainly UV radiation. It also inhibits a specific DNA repair system as well as alters immune system (e.g. by affecting mast cells) and thus leading to deregulation of several important immune responses. Apart from this, arsenic was also reported to enhance keratinocytes to express factors that may further induce cutaneous tumorigenesis, such as TGF- $\alpha$, IL-6 or IL-8 [40, 41]. Gossai et al. carried out a population-based case-control study of 487 SCC cases and 462 age- and gendermatched controls in the United States. The authors found evidence that rice consumption may be related to the occurrence of SCC of the skin (95\% Cl: 1.1-2.0), and the association may be stronger in those with low arsenic exposure from drinking water [42]. Rice cultivars vary widely in their ability to accumulate As, but brown rice contains more As than white rice because inorganic arsenic concentrates in the outer layer of brown husks. This, in theory, could explain the higher risk of SCC observed with brown rice consumption opposite to white rice.

Very interesting results from the original paper by Bojkova et al. presented that melatonin, as a dietary factor, could modulate a cancer risk when used with certain 
Table 1. Summary of cutaneous squamous cell carcinoma (cSCC) risk factors

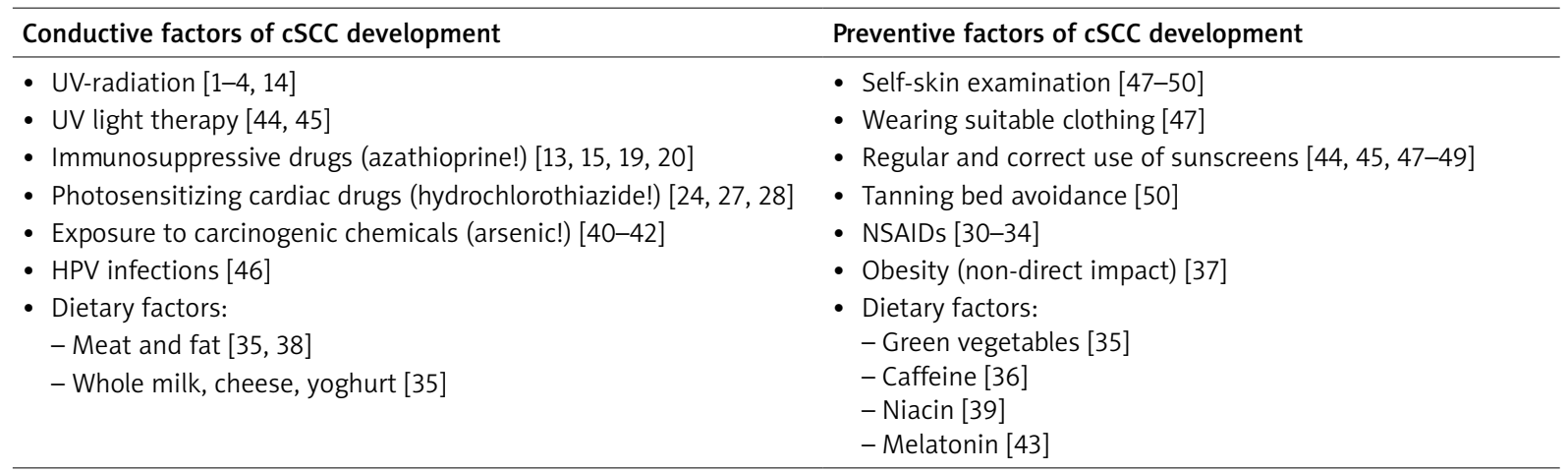

drugs which include non-steroidal anti-inflammatory drugs, statins and antidiabetics [43]. Melatonin is contained in meat (lamb, beef, pork), fish (salmon), chicken, nuts, bananas, tomatoes, rice, cucumber and beetroot. The authors suggest that intake of these dietary components could possibly increase melatonin in humans resulting in prevention of SCC carcinogenesis. The concentration of melatonin was found to be higher in fish sources, for example in salmon - $3.7 \mathrm{ng} / \mathrm{g}$ and eggs $6.1 \mathrm{ng} / \mathrm{g}$, compared to other animal products including beef $-2.2 \mathrm{ng} / \mathrm{g}$ and pork - $2.5 \mathrm{ng} / \mathrm{g}$ [43].

To summarize, leafy vegetables, nuts, fish, not fat meet, caffeine, niacin are preventive factors for cutaneous squamous cell cancer. It is recommended to exercise and eat regular meals containing a variety of vitamins, minerals and bioactive substances such as antioxidants, vitamins $\mathrm{C}$ and $\mathrm{E}$, flavonoids, fibre and folic acid.

\section{Conclusions}

SCC of the skin is the second most common nonmelanoma skin neoplasm. Cutaneous SCC may occur on any part of the body, but the most common site is skin chronically exposed to UV radiation, such as head and neck (including lower lip and ears) comprising more than half of all cases of SCCS, followed by forearms and the dorsum of the hands. CSCC constitutes the most common skin cancer capable of metastasis formation [1]

The aetiology includes exogenous risk factors such as sunlight exposure, HPV infection, immunosuppressive and cardiac photosensitizing drugs, influence of carcinogens like arsenic and some dietary components. The risk of cSCC development depends on a lifetime accumulation of UV radiation. Therefore, the reduction of the ozone layer and ageing population are related to rising incidence of cSCC worldwide.

Chlorine containing drugs, such as hydrochlorothiazide, chloroquine, furosemide, amiloride and diclofenac exhibit photochemical activity leading to free radical reactions with lipids, proteins and DNA responsible for their damage. In concert with this mechanism, some recent studies suggest that diet supplementation with an- tioxidants may be beneficial in increasing the minimum erythemal UV radiation dose.

Particularly thorough attention should be paid to immunocompromised patients, who present the highest incidence of SCC morbidity and mortality. Certain immunosuppressive drugs, i.e. azathioprine and calcineurin inhibitors, increase this risk more than others. Therefore, frequently performed skin check-ups provide the main protection and early skin cancer detection in this SCC severely-affected group. In cardiology, great attention should be paid to early change of cardiac photosensitizing antihypertensive drugs to non-photosensitizing ones.

Each method of SCC prevention including diet supplementation deserves attention and the analysis of its possible usefulness if proved effective. The main preventive factors of SCC development are included in Table 1. Despite a multitude of different therapeutic methods of CSCC treatment, the diversity of SCC considerably reduces the effectiveness of a single method. The knowledge regarding the most significant risk factors may lead to successful prevention and reduction of incidence of cutaneous SCC. Public education campaigns should promote skin self-examination and emphasize the importance of photoprotection, tanning bed avoidance, early skin cancer detection and treatment.

\section{Conflict of interest}

The authors declare no conflict of interest.

\section{References}

1. Lesiak A, Czuwara J, Kamińska-Winciorek G, et al. Squamous cell carcinoma and Merkel-cell carcinoma. Diagnostic and therapeutic recommendations of the Polish Dermatological Society. Dermatol Rev 2019; 106: 127-49.

2. Piacentini RD, Della Ceca LS, Ipińa A. Climate change and its relationship with non-melanoma skin cancers. Photochem Photobiol Sci 2018; 17: 1913-7.

3. Voiculescu V, Calenic B, Ghita M, et al. From normal skin to squamous cell carcinoma: a quest for novel biomarkers. Dis Markers 2016; 2016: 4517492.

4. Armstrong BK, Kricker AJ. The epidemiology of UV induced skin cancer. Photochem Photobiol B 2001; 63: 8-18. 
5. Pandeya N, Olsen CM, Whiteman DC. The incidence and multiplicity rates of keratinocyte cancers in Australia. Med J Aust 2017; 207: 339-43.

6. Muzic JG, Schmitt AR, Baum CL, et al. Incidence and trends of basal cell carcinoma and cutaneous squamous cell carcinoma: a population-based study in Olmsted County, Minnesota, 2000 to 2010. Mayo Clin Proc 2017; 92: 890-8.

7. Cheng JY, Li FY, Ko CJ, et al. Cutaneous squamous cell carcinomas in solid organ transplant recipients compared with immunocompetent patients. JAMA Dermatol 2018; 154: 60-6.

8. Greenberg JN, Zwald FO. Management of skin cancer in solid organ transplant recipients: a multidisciplinary approach. Dermatol Clin 2011; 29: 231-41.

9. Moloney FJ, Comber H, O'Lorcain P, et al. A population-based study of skin cancer incidence and prevalence in renal transplant recipients. Br J Dermatol 2006; 154: 498-504.

10. Collett D, Mumford L, Banner NR, et al. Comparison of the incidence of malignancy in recipients of different types of organ: a UK Registry audit. Am J Transplant 2010; 10: 1889-96.

11. Lindelöf B, Granath F, Dal H, et al. Sun habits in kidney transplant recipients with skin cancer: a case-control study of possible causative factors. Acta Derm Venerol 2003; 83: 189-93.

12. Molina BD, Leiro MGC, Pulpon LA, et al. Incidence and risk factors for nonmelanoma skin cancer after heart transplantation. Transplant Proc 2010; 42: 3001-5.

13. Cho HG, Kuo KY, Xiao K, et al. Azathioprine and risk of multiple keratinocyte cancers. J Am Acad Dermatol 2018; 78 : 27-8.e1.

14. Atkar R, Okampo M, Euvrard S, et al. Ultraviolet radiation exposure through window glass may be associated with localization of non-melanoma skin cancer in organ transplant recipients: a study in France and the UK. Br J Dermatol 2013; 169: 484-5.

15. Vos M, Plasmeijer El, van Bemmel BC, et al. Azathioprine to mycophenolate mofetil transition and risk of squamous cell carcinoma after lung transplantation. J Heart Lung Transplant 2018; 37: 853-9.

16. Secnikova Z, Gopfertova D, Hoskova L, et al. Significantly higher incidence of skin cancer than other malignancies in patients after heart transplantation. A retrospective cohort study in the Czech Republic. Biomed Pap Med Fac Univ Palacky Olomouc Czech Repub 2015; 159: 648-51.

17. Sobieszczańska-Małek M, Komuda K, Piotrowska M, et al. Incidence of malignancies in cardiac allograft recipients a single-center experience. Ann Transplant 2013; 18: 88-94.

18. Jäämaa-Holmberg S, Salmela B, Lemström K, et al. Cancer incidence and mortality after heart transplantation - a population-based national cohort study. Acta Oncol 2019; 58: 859-63.

19. Na R, Laaksonen MA, Grulich AE, et al. High azathioprine dose and lip cancer risk in liver, heart, and lung transplant recipients: a population-based cohort study. J Am Acad Dermatol 2016; 74: 1144-52.

20. Robson RA, Opelz G, Cecka JM, et al. Risk of PTLD and other malignancies in renal transplant patients treated with MMF: a prospective observational cohort study. Am J Transplant 2003; 3 Suppl 5: 187.

21. De Rosa N, Paddon VL, Liu Z, et al. Nonmelanoma skin cancer frequency and risk factors in Australian heart and lung transplant recipients. JAMA Dermatol 2019; 155: 716-9.

22. Albayati A, Ozkan B, Eyuboglu AA, et al. Nonmelanoma skin cancers in solid-organ transplant recipients: a single center experience. Exp Clin Transplant 2018; 16 Suppl 1: 95-100.
23. Carroll RP, Segundo DS, Hollowood K, et al. Immune phenotype predicts risk for posttransplantation squamous cell carcinoma. J Am Soc Nephrol 2010; 21: 713-22.

24. Pottegård A, Hallas J, Olesen M, et al. Hydrochlorothiazide use is strongly associated with risk of lip cancer. J Intern Med 2017; 282: 322-31.

25. Su KA, Habel LA, Achacoso NS, et al. Photosensitizing antihypertensive drug use and risk of cutaneous squamous cell carcinoma. Br J Dermatol 2018; 179: 1088-94.

26. Wang YR. Outpatient hypertension treatment, treatment intensification, and control in Western Europe and the United States. Arch Intern Med 2007; 167: 141-7.

27. Pedersen SA, Gaist D, Schmidt SAJ, et al. Hydrochlorothiazide use and risk of nonmelanoma skin cancer: a nationwide case-control study from Denmark. J Am Acad Dermatol 2018; 78: 673-81.

28. Shin D, Lee ES, Kim J, et al. Association between the use of thiazide diuretics and the risk of skin cancers: a meta-analysis of observational studies. J Clin Med Res 2019; 11: 247-55.

29. Nardone B, Majewski S, Kim AS, et al. Melanoma and nonmelanoma skin cancer associated with angiotensin-converting-enzyme inhibitors, angiotensin-receptor blockers and thiazides: a matched cohort study. Drug Saf 2017; 40: 249-55.

30. Johannesdottir SA, Chang ET, Mehnert F, et al. Nonsteroidal anti-inflammatory drugs and the risk of skin cancer: a population-based case-control study. Cancer 2012; 118: 4768-76.

31. Reinau D, Surber C, Jick SS, et al. Nonsteroidal anti-inflammatory drugs and the risk of nonmelanoma skin cancer. Int J Cancer 2015; 137: 144-53.

32. Muranushi C, Olsen CM, Pandeya N, et al. Aspirin and nonsteroidal anti-inflammatory drugs can prevent cutaneous squamous cell carcinoma: a systematic review and metaanalysis. J Invest Dermatol 2015; 135: 975-83.

33. Wysong A, Ally MS, Gamba CS, et al. Non-melanoma skin cancer and NSAID use in women with a history of skin cancer in the Women's Health Initiative. Prev Med 2014; 69: 8-12.

34. Pandeya N, Olsen CM, Thompson BS, et al. Aspirin and nonsteroidal anti-inflammatory drug use and keratinocyte cancers: a large population-based cohort study of skin cancer in Australia. Br J Dermatol 2019; 181: 749-60.

35. Hughes MC, Van Der Pols JC, Marks GC, et al. Food intake and risk of squamous cell carcinoma of the skin in a community: the Nambour skin cancer cohort study. Int J Cancer 2006; 119: 1953-60.

36. Abel EL, Hendrix SO, McNeeley SG, et al. Daily coffee consumption and prevalence of nonmelanoma skin cancer in Caucasian women. Eur J Cancer Prev 2007; 16: 446-52.

37. Pothiawala S, Qureshi AA, Li Y, et al. Obesity and the incidence of skin cancer in US Caucasians. Cancer Causes Control 2012; 23: 717-26.

38. Ibiebele TI, van der Pols JC, Hughes MC, et al. Dietary fat intake and risk of skin cancer: a prospective study in Australian adults. Int J Cancer 2009; 125: 1678-84.

39. Park SM, Li T, Wu S, et al. Niacin intake and risk of skin cancer in US women and men. Int J Cancer 2017; 140: 2023-31.

40. Gundert-Remy U, Damm G, Foth H, et al. High exposure to inorganic arsenic by food: the need for risk reduction. Arch Toxicol 2015; 89: 2219-27.

41. Vega L, Styblo M, Patterson R, et al. Differential effects of trivalent and pentavalent arsenicals on cell proliferation and cytokine secretion in normal human epidermal keratinocytes. Toxicol Appl Pharmacol 2001; 172: 225-32. 
42. Gossai A, Zens M, Punshon T, et al. Rice consumption and squamous cell carcinoma of the skin in a United States Population. Environ Health Perspect 2017; 125: 097005.

43. Bojková B, Kubatka P, Qaradakhi T, et al. Melatonin may increase anticancer potential of pleiotropic drugs. Int J Mol Sci 2018; 19: 3910.

44. Stern RS. The risk of squamous cell and basal cell cancer associated with psoralen and ultraviolet A therapy: a 30-year prospective study. J Am Acad Dermatol 2012; 66: 553-62.

45. Raone B, Patrizi A, Gurioli C, et al. Cutaneous carcinogenic risk evaluation in 375 patients treated with narrowband UVB phototherapy: a 15-year experience from our Institute. Photodermatol Photoimmunol Photomed 2018; 34: 302-6.

46. Quint KD, Genders RE, de Koning MN, et al. Human Betapapillomavirus infection and keratinocyte carcinomas. J Pathol 2015; 235: 342-54.

47. Calzavara-Pinton P, Ortel B, Venturini M. Non-melanoma skin cancer, sun exposure and sun protection. G Ital Dermatol Venereol 2015; 150: 369-78.

48. Leiter U, Eigentler T, Garbe C. Epidemiology of skin cancer. Adv Exp Med Biol 2014; 810: 120-40.

49. John SM, Trakatelli M, Gehring R, et al. Consensus Report: Recognizing non-melanoma skin cancer, including actinic keratosis, as an occupational disease - a call to action. J Eur Acad Dermatology Venereol 2016; 30: 38-45.

50. Wehner MR, Shive ML, Chren MM, et al. Indoor tanning and non-melanoma skin cancer: systematic review and metaanalysis. BMJ 2012; 345: e5909. 